\title{
High thermosensitivity of silicon nanowires induced by amorphization
}

\author{
Toan Dinh ${ }^{\mathrm{a}}$, Hoang-Phuong Phan ${ }^{\mathrm{a}}$, Takahiro Kozeki ${ }^{\mathrm{c}}$, Afzaal Qamar ${ }^{\mathrm{a}}$, Tatsuya Fujii ${ }^{\mathrm{c}}$, Takahiro \\ $\mathrm{Namazu}^{\mathrm{c}}$, Nam-Trung Nguyen ${ }^{\mathrm{a}}$, Dzung Viet Dao ${ }^{\mathrm{a}, \mathrm{b}}$ \\ ${ }^{a}$ Queensland Micro- and Nanotechnology Centre, Griffith University, Queensland, Australia \\ ${ }^{b}$ Griffith School of Engineering, Griffith University, Queensland, Australia \\ ${ }^{c}$ Department of Mechanical Engineering, University of Hyogo, Hyogo, Japan
}

\begin{abstract}
In this work, we demonstrate highly thermosensitive silicon nanowires (SiNWs) for thermal-sensing applications. Crystalline Si was amorphized by Focused Ion Beam in the fabrication process of the SiNWs, and subsequently recrystallized by a thermal annealing process to improve their electrical conductivity. A temperature coefficient of resistance (TCR) from $-8,000 \mathrm{ppm} / \mathrm{K}$ to $-12,000 \mathrm{ppm} / \mathrm{K}$ was measured for the SiNWs. This large negative TCR is attributed to the boundary potential barrier of 110 meV between silicon crystallites in the poly crystalline SiNWs.

Keywords: Thermoresistive effect, temperature coefficient of resistance, silicon nanowire, amorphization, focused ion beam.

$2015 M S C$ :
\end{abstract}

\section{Introduction}

Over the past several decades, electronic sensing devices have been significantly developed, with an emphasis on miniaturization and high sensitivity with various successful applications [1, 2, 3, 4, 5]. Nanowires (NWs), as an important achievement of the miniaturization process, are obviously of interest, since these one-dimensional nanostructures offer extremely small sizes with a great integration ability into micro/nano systems [6, 7]. For instance, recent research facilitating thermal-sensing nano-systems has focused on highly thermosensitive NWs which are particularly useful for monitoring temperature variations within a narrow range [8].

To estimate the themosensitivity of NWs, temperature coefficient of resistance (TCR) has been widely employed and can be defined as the relative change of resistance to the temperature variation [9]. For example, metal nanowires showed a positive TCR ranging from 2500 to $4000 \mathrm{ppm} / \mathrm{K}$ [10], which was similar to that of bulk metals [11. In addition, the TCR of semiconductive nanowires can be controlled through doping levels, but it has been shown to be relatively low and in a limited range

\footnotetext{
* Corresponding author

Email address: toan.dinh@griffithuni.edu.au (Toan Dinh)
} 
[12, 13] (-3700 to $700 \mathrm{ppm} / \mathrm{K})$. Apart from doping, morphologies such as crystalline and amorphous could also vary the thermal properties of a material because the TCR significantly depends on the number of defects and grain boundaries in the crystallographic structures [14, 15].

It is well known that Focused Ion Beam (FIB) is a common technique which damages the crystallites to form amorphous nanostructures [16, 17. Moreover, thermal annealing is a simple approach for recrystallizing the damaged material and improving its electrical conductivity for ease of measurements [18. In fact, the thermal annealing method has also been utilized to improve the quality of solar cell [19], the conductivity and stability of transparent electrodes/heaters 20], and the properties of other electronic devices [18, 20]. Therefore, NWs, fabricated by FIB and a subsequent thermal annealing, could be a suitable solution for developing highly-sensitive thermal-sensing nanosystems.

In this study, we fabricated silicon nanowires (SiNWs) from a Silicon on Insulator (SOI) wafer using FIB, wet etching and subsequent annealing processes, and observed an extremely large negative TCR for the SiNWs $(-8,000 \mathrm{ppm} / \mathrm{K}$ to $-12,000 \mathrm{ppm} / \mathrm{K})$. We also investigated the change in the morphologies of the SiNWs with the FIB and the annealing process, and discussed the conduction mechanism of the highly thermosensitive SiNWs.

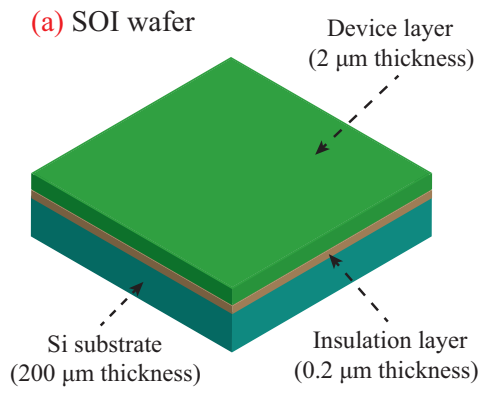

(c) Wet etching

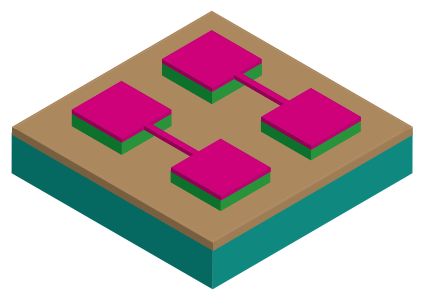

n-type Si substrate $\mathrm{SiO}_{2}$ layer (b) FIB implantation

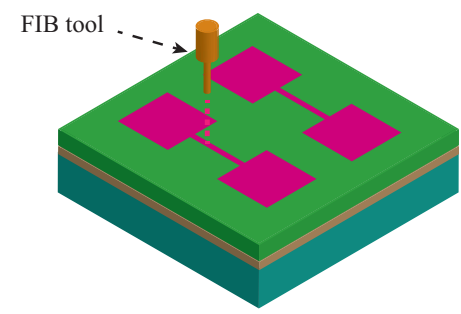

(d) SEM image of a SiNW

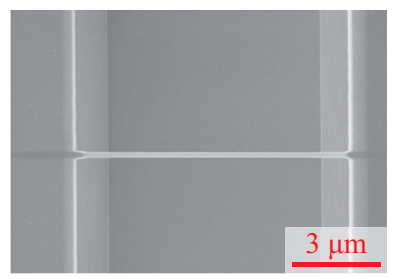

Single crystalline $\mathrm{Si}$

Amorphized Si

Figure 1: Fabrication of SiNWs: (a) Silicon on Insulator (SOI) wafer; (b) $\mathrm{Ga}^{+}$implantation using FIB; (c) Wet etching to form the SiNWs; (d) Scanning Electron Microscropy (SEM) image of a SiNW. 


\section{Fabrication of SiNWs} shown in Fig. 1(a). The device, insulation and substrate layers are highly doped single crystalline $\mathrm{Si}$, silicon dioxide and low doped crystalline $\mathrm{Si}$, respectively. At the FIB step, an implantation of gallium ions (TMHitachi FB2200, voltage $40 \mathrm{kV}$, beam current $110 \mathrm{pA}$ ) was employed with a dose of approximately $7 \times 10^{15}$ ions $/ \mathrm{cm}^{2}$ to damage the crystalline $\mathrm{Si}$ areas and form amorphous morphology dimensions of $0.2(0.3) \mu m \times 10 \mu m$. It is noted that with the above FIB conditions, the thickness of the implanted layer was measured to be approximately $60 \mathrm{~nm}$.

In the next step, the SiNWs were released using a wet etching process with tetramethylammonium hydroxide hydroxide (TMAH, $20 \mathrm{wt} \%$ ) at $90{ }^{\circ} \mathrm{C}$, owing to the low etching rate of the amorphous $\mathrm{Si}$ at a temperature of $700{ }^{\circ} \mathrm{C}$ for 60 minutes in a high-vacuum chamber, to improve their electrical conductivity for electrical measurements. It is expected that increasing the annealing temperatures could reduce the number of boundaries and defects and enhance the crystallite size [21]. On the other hand, at low annealing temperatures, the improvement of electrical conductivity of the SiNWs might

1(d) shows a scanning electron microscope (SEM) image of a fabricated SiNW with a dimension of 10 $\mu \mathrm{m}$ length, $300 \mathrm{~nm}$ width and $60 \mathrm{~nm}$ thickness.

\section{Results and discussion}

\subsection{Characterization of SiNW morphology}

The impact of the fabrication process on the morphology of the implanted Si layer was investigated by Raman measurements (Fig. 2(a)), which indicates a sharp peak (at a wavenumber of $520 \mathrm{~cm}^{-1}$ ), no peak and a moderate peak for the layer before FIB, after FIB and after annealing processes, respectively. This effect implies a transformation in morphology with the FIB and annealing processes. The change of the layer morphology was confirmed and is shown in Figure 2(b) and (c), which indicates the disappearance of the boundary between the damaged layer and the single crystalline layer. The high-resolution transmission electron microscopy (HRTEM) image (Figure2(d)) shows the amorphous structure of an implanted area after FIB. To confirm the amorphous property of the implanted Si layer, the selected area electron diffraction (SAED) measurements were performed on a small area which included point A, as shown in the inset Figure 2(d). The broad and diffuse rings in the SAED image are clearly observed. This is a well-known evidence for the amorphous characteristic of the implanted film 22]. This amorphization is attributed to the fact that $\mathrm{Ga}+$ ions collided with the $\mathrm{Si}$ 

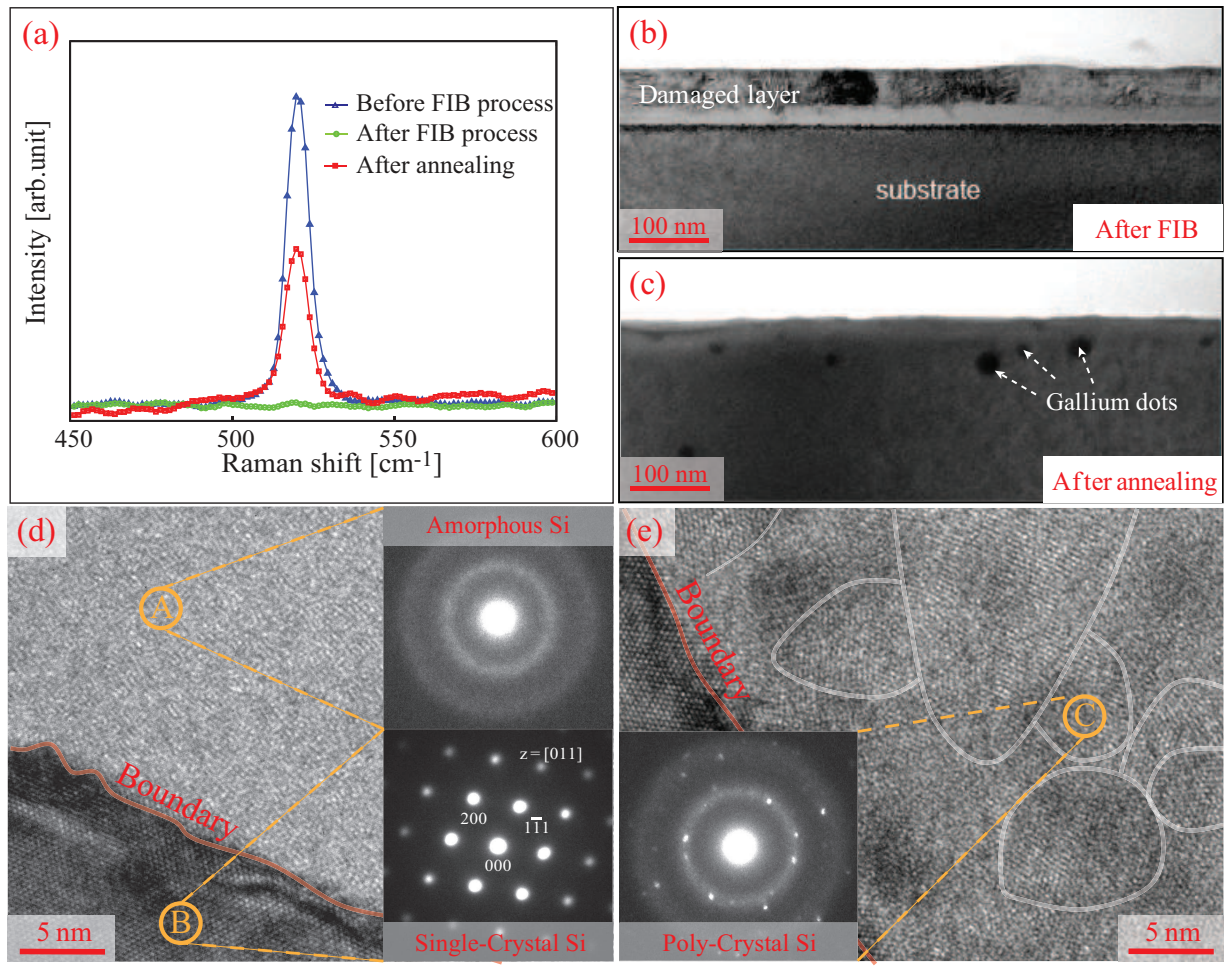

Figure 2: Impact of the fabrication process on the morphology and Raman spectrum of the implanted Si layer: (a) Raman spectrum; (b,c) Transmission electron microscopy (TEM) images of the damaged layer (b) after FIB and (c) after annealing; (d,e) High-resolution transmission electron microscopy (HRTEM) images show the morphology of the implanted Si layer (d) after FIB and (e) after annealing. [(d,e) Reproduced from Ref. 16 with permission from the Royal Society of Chemistry].

atoms during the FIB implantation, leading to the damage of Si crystals. With a large Ga+ dose of $7 \times 10^{15} \mathrm{~cm}^{-2}$, the damaged layer became amorphous Si. The SAED image of point B indicates the single crystalline patterns of the unimplanted Si layer (inset Fig. 2(d)). The HRTEM image (Fig. 2(e)) shows the poly crystalline structure of the Si layer after the annealing process. The poly crystalline was confirmed by SAED measurements around point C (Inset Fig. 2(e)) with small spots forming the rings. The thermal annealing allowed the $\mathrm{Si}$ atoms to occupy lattice the sites, and exclude the Ga+ impurities [23, resulting in the recrystallization of the amorphized Si layer.

\subsection{Thermoresistive properties of the SiNWs}

Figure 3(a) shows the current-voltage characteristics of 200 and $300 \mathrm{~nm}$ SiNWs, which illustrates an increase in the electrical conductivity at least one order of magnitude (approximately from 0.007 to 0.14 $\mathrm{Scm}^{-1}$ ) after the annealing process. This also is evident for the recrystallization of the amorphous SiNWs, in which the number of defects and boundaries reduces along with an increase in carrier concentration and carrier mobility. 

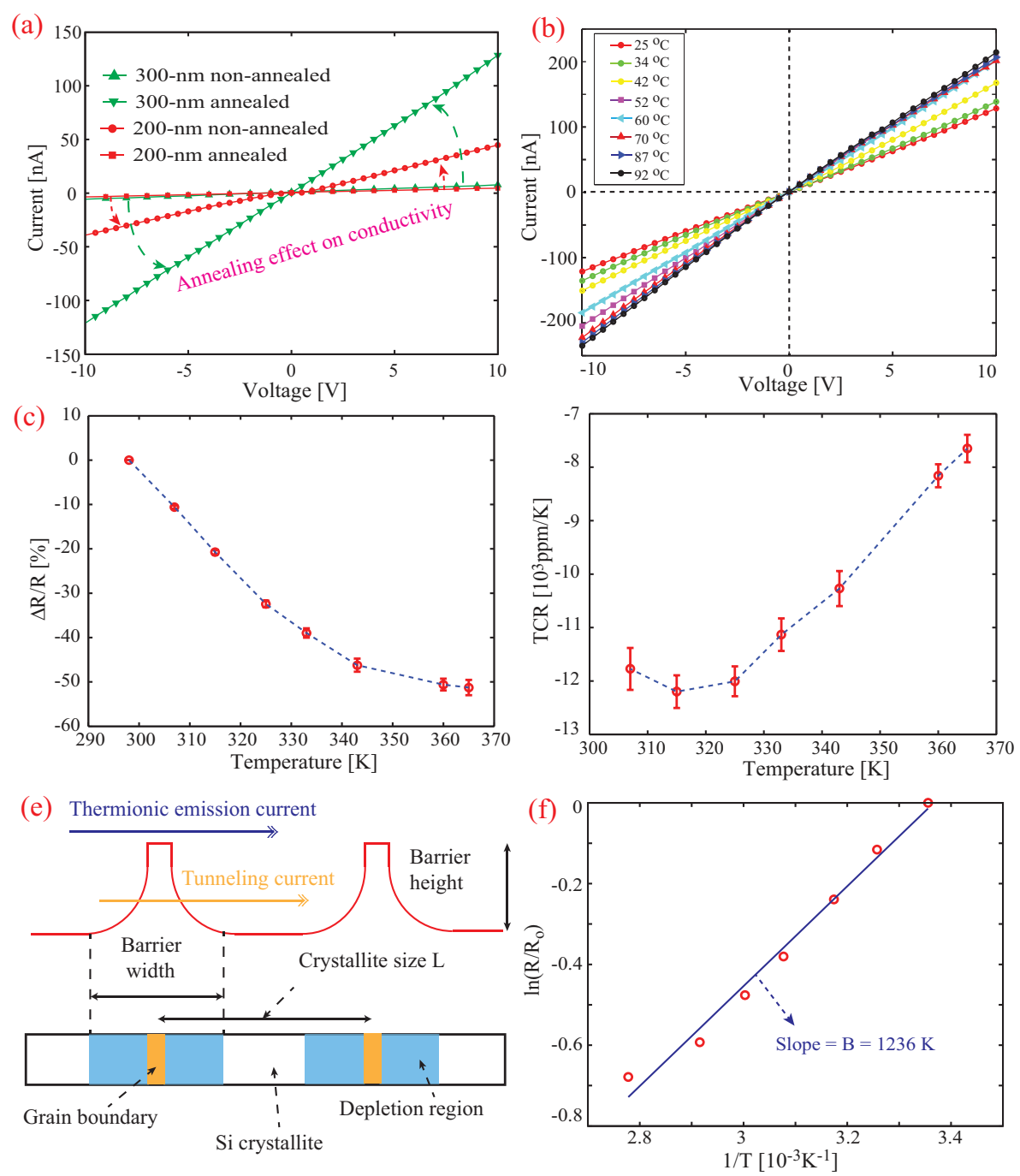

Figure 3: Thermoresistive characteristics of the SiNWs: (a) Annealing effect on the conductivity of the SiNWs; (b) Temperature-dependent I-V characteristics of the SiNWs; (c) Relative resistance change; (d) Temperature coefficient of resistance (TCR); (e) Schematic sketch of Si crystallites and potential barriers at their boundaries; (f) Arrhenius plot of SiNW thermoresistance (the slope shows the thermal index of $1236 \mathrm{~K}$ ).

Figure 3(b) shows the typical current-voltage characteristics of the SiNWs. The measured currents increased with increasing temperature, which indicated that the conduction of the SiNW was thermally activated. The strongly temperature-dependent thermoresistive properties of the SiNW are shown in Fig. 2(c) with a decrease of more than $50 \%$ at a temperature change of $70{ }^{\circ} \mathrm{C}$. Correspondingly, the negative temperature coefficient of resistance (TCR) was found to be extremely large, ranging from $-8,000 \mathrm{ppm} / \mathrm{K}$ to $-12,000 \mathrm{ppm} / \mathrm{K}$ (Fig. 3(d)). This TCR value is much larger than that of various SiNWs reported in the literature [12, 13. The high thermosensitivity of the SiNWs is attributed to 
the morphology of the implanted and incompletely recrystallized Si. As such, poly/nano crystalline structures typically have a number of defects and boundaries, which can trap the free carriers and deplete the adjacent regions of the crystallites. This leads to a certain portion of dopant to be inactive and results in formation of a potential barrier $\phi$ which impedes the movement of the carriers between crystallites (Fig. 3(e)). When the temperature increases, thermionic emission of energetic carriers over the barrier and tunnelling of the carriers through the barrier cause a significant decrease in the electrical resistivity of the SiNWs [15]. In other words, the high thermosensitivity was obtained for the SiNWs. Moreover, as a very low carrier concentration $\left(2 \times 10^{16} \mathrm{~cm}^{-3}\right)$ was estimated for the as-fabricated SiNWs [17, only a portion of impurities is ionized at room temperature [9]. When the temperature increases, more thermally excited carriers are generated, which leads to the increase in the electrical conductivity of the SiNWs.

As observed by the Raman, TEM and HRTEM measurements, the implanted Si layer had a poly crystalline structure after the annealing process. Therefore, the electrical resistance of the SiNWs is assumed to come from the resistance of the crystallites and their boundary resistance. Because of the fact that the Si grain resistance is much smaller than the boundary resistance [15, the electrical resistance $R$ of the SiNWs can be approximated as the boundary resistance, which is written in the following form [14]:

$$
R \sim \exp \left(\frac{\phi}{k T}\right)
$$

where $k$ is the Boltzmann constant and $T$ is the absolute temperature. Therefore, the relationship between the electrical resistance of the SiNWs and the temperature can be expressed as follows:

$$
R=A \exp \left(\frac{B}{T}\right)
$$

where $A$ is a constant, and $B=\phi / k$ is the thermal index of the SiNWs. Eq. 2 can also be rewritten in the following form:

$$
\ln \left(R / R_{0}\right)=B\left(\frac{1}{T}-\frac{1}{T_{0}}\right)
$$

where $R_{0}$ is the resistance at the reference temperature $T_{0}$. From the slope of Arrhenius plot (Fig. $3(\mathrm{f})$ ), the thermal index was found to be $1236 \mathrm{~K}$, which is comparable to that of various highly sensitive thermistors reported in literature [24, 25]. This indicates the strong feasibility of using the SiNWs for highly thermosensitive nano-devices.

Since the thermal index of the SiNWs was estimated, the potential barrier was found to be approximately $110 \mathrm{meV}$. This potential value is quite large in comparison with that of the highly doped polycrystalline Si reported in [15]. When the temperature increases, the carriers could be excited by 
the thermal energy, thus they could possess high enough energy to surmount the potential barrier at the grain boundary [15]. The movement of the thermally excited carriers results in an electrical current which is thermionic emission current (Fig. 3(e)). It is noted that this transport mechanism has been widely employed for highly doped polysilicon because the potential barrier is relatively low with high doping levels, and the thermally excited carriers needs a small amount of energy to pass over the barrier [15. However, as the implanted SiNWs have a low carrier concentration $\left(2 \times 10^{16} \mathrm{~cm}^{-3}\right)$, the potential barrier was found to be relatively high $(110 \mathrm{meV})$. Therefore, carriers with energy less than this barrier could only go through this barrier by quantum-mechanic tunnelling [15]. When the barrier is high enough, the tunnelling current could become much larger than the thermionic emission current. Consequently, the quantum-mechanic tunnelling mechanism could be an important factor for the significant decrease in the electrical resistance of the SiNWs with increasing temperature [14, 15].

\section{Conclusion}

In conclusion, we fabricated highly thermosensitive SiNWs using a FIB implantation and a subsequent thermal annealing process. The impact of the fabrication process on the morphology of the implanted Si was investigated. A large negative TCR of the SiNWs of up to $-12,000 \mathrm{ppm} / \mathrm{K}$ was found, which is attributed to a high potential barrier of $110 \mathrm{meV}$ between silicon crystallites. These results indicated the possibility of using the SiNWs for highly thermosensitive nano-sensors.

\section{Acknowledgments}

This work was performed in part at the Queensland node of the Australian National Fabrication Facility, a company established under the National Collaborative Research Infrastructure Strategy to provide nano and micro-fabrication facilities for Australia's researchers.

\section{References}

[1] Kolmakov A., Zhang Y., Cheng G. and Moskovits M., Advanced Materials, 2003, 15(12), 997-1000.

[2] Chung S.W., Yu J.Y. and Heath J.R., Applied Physics Letters, 76, 2068 (2000).

[3] Dau V. T., Dao D. V., Yamada T., Tung B. T., Hata K., and Sugiyama S., Smart Materials and structures, 2010, 19(7), 075003.

[4] Dao D. V., Nakamura K., Bui T. T. and Sugiyama S., Advances in Natural Sciences: Nanoscience and Nanotechnology,2010, 1(1), 013001. 
[5] Dao D. V., Toriyama T., Wells J. and Sugiyama S., 2002, In the Fifteenth IEEE International Conference on Micro Electro Mechanical Systems, 2012, 312-315.

[6] Whang, D., Jin, S., Wu, Y. and Lieber, C.M., Nano letters, 2003, 3(9), 1255-1259.

[7] Zhong, Z., Wang, D., Cui, Y., Bockrath, M.W. and Lieber, C.M., Science, 2003, 302(5649), 13771379.

[8] Yang, Y., Zhou, Y., Wu, J.M. and Wang, Z.L., ACS nano, 2012, 6(9), 8456-8461.

[9] Dinh T., Phan H.P., Kozeki T., Qamar A., Namazu T., Nguyen N.T. and Dao D.V., RSC Advances, 2015, 5(128), 106083-106086.

[10] Bid, A., Bora, A., and Raychaudhuri, A. K. Physical Review B: Condensed Matter and Materials Physics, 2006, 74(3), 035426.

[11] Kuo J. T. W., Yu L., and E. Meng. Micromachines, 2012, 3, 550-573.

[12] Wang, C. P., Liu, C. W., and Gau, C. In Nano/Micro Engineered and Molecular Systems (NEMS), IEEE International Conference on, February 2011, pp. 630-633.

[13] Bosseboeuf A., Allain P. E., Parrain F., Le Roux X., Isac N., Jacob S., and Walther A., Advances in Natural Sciences: Nanoscience and Nanotechnology, 2015, 6(2), 025001.

[14] Dinh T., Phan H.P., Dao D.V., Woodfield P., Qamar A. and Nguyen N.T., Journal of Materials Chemistry C, 2015, 3(34), 8776-8779.

[15] Seto J. Y., Journal of Applied Physics, 46(12), 5247-5254, 1975.

[16] Brugger J., Beljakovic G., Despont M., De Rooij N. F., and Vettiger P., Microeleetronic Engineering, 1997, 35(LMIS1-ARTICLE-2005-015), 401-404.

[17] Phan H. P., Kozeki T., Dinh T., Fujii T., Qamar A., Zhu Y., Namazu T., Nguyen N. T. and Dao D. V., RSC Advances, 2015, 5(100), 82121-82126.

[18] Nakamura S., Mukai T., Senoh M. and Iwasa N., Japanese Journal of Applied Physics, 1992, 31(2B), L139.

[19] Zang Z., Nakamura A. and Temmyo J., Optics express, 2013, 21(9), 11448-11456.

[20] Langley D.P., Lagrange M., Giusti G., Jimenez C., Brechet Y., Nguyen N.D. and Bellet D., Nanoscale, 2014, 6(22), 13535-13543.

[21] Xiao Y.J., Fang F.Z., Xu Z.W. and Hu X.T., Applied Surface Science, 2015, 343, 56-69. 
[22] Yan H.F., Xing Y.J., Hang Q.L., Yu D.P., Wang Y.P., Xu J., Xi Z.H. and Feng S.Q., Chemical Physics Letters, 2000, 323(3), 224-228.

[23] Jaeger R. C., Introduction to Microelectronic Fabrication, Prentice Hall, New Jersey, 2nd edn, 2001.

[24] Dinh T., Dao D. V., Phan H. P., Wang L., Qamar A., Nguyen N. T., and Rybachuk M., Applied Physics Express, 2015, 8(6), 061303.

[25] Kong D., Le L.T., Li Y., Zunino J.L. and Lee W., Langmuir, 2012, 28(37), 13467-13472. 